\title{
Revista Colombiana de

\section{Eficacia y seguridad de la atorvastatina en dosis altas en pacientes con enfermedad renal crónica estadio 5 en terapia de diálisis peritoneal}

\author{
César A. Restrepo ${ }^{a, *}$ y Jesús A. Castaño ${ }^{b}$ \\ a Servicio de Medicina Interna-Nefrología, Universidad de Caldas, Manizales, Colombia \\ b Servicio de Medicina Interna, Universidad de Caldas, Manizales, Colombia
}

Recibido el 20 de febrero de 2014; aceptado el 12 de mayo de 2014

Disponible en Internet el 17 de diciembre de 2014

\section{PALABRAS CLAVE \\ Colesterol; \\ Estatinas; \\ Hipercolesterolemia; \\ Riñón}

\begin{abstract}
Resumen
Objetivo: Determinar cambios en el perfil lipídico y seguridad del tratamiento con atorvastatina en dosis altas (mayor o igual a $40 \mathrm{mg} /$ día) en pacientes con enfermedad renal crónica estadio 5.

Pacientes, materiales y métodos: Pacientes con enfermedad renal crónica estadio 5 en terapia de diálisis peritoneal e hipercolesterolemia, refractarios a terapia con lovastatina en dosis de $40 \mathrm{mg}$ al día y atorvastatina (menos de $40 \mathrm{mg} /$ día), a quienes se les asignó terapia con atorvastatina en dosis altas.

Análisis estadístico: Estudio longitudinal, retroprospectivo, correlacional, unicéntrico con análisis univariado y bivariado.

Resultados: Veinte pacientes cumplieron con los criterios de inclusión. El tiempo con atorvastatina en dosis altas fue en promedio de 27 meses. La dosis promedio utilizada fue $60,4 \mathrm{mg} /$ día. La tendencia beneficiosa a lograr metas terapéuticas con las variables en el tiempo se logró en el $65 \%$ de los pacientes para colesterol total, $50 \%$ para colesterol LDL, $40 \%$ para colesterol no-HDL y $45 \%$ para triglicéridos. Para las variables colesterol LDL y colesterol HDL se encontró diferencia estadísticamente significativa entre los valores iniciales y finales $(p<0,05)$. Se documentaron 26 complicaciones, ninguna de ellas considerada un evento adverso relacionado con la administración de la atorvastatina en dosis altas.

Conclusiones: En pacientes con enfermedad renal crónica estadio 5, mayores de 50 años, en terapia de diálisis peritoneal e hipercolesterolemia, la atorvastatina en dosis altas logra obtener niveles de LDL en metas en el $50 \%$ de los pacientes tratados, sin que su administración dé lugar a eventos adversos importantes.

(c) 2014 Sociedad Colombiana de Cardiología y Cirugía Cardiovascular. Publicado por Elsevier España, S.L.U. Todos los derechos reservados.
\end{abstract}

\footnotetext{
* Autor para correspondencia.

Correo electrónico: caugustorv@une.net.co (C.A. Restrepo).
} 


\section{KEYWORDS}

Cholesterol;

Statins;

Hypercholesterolemia; Kidney
Efficacy and safety of high-dose atorvastatin in patients with stage 5 chronic kidney disease on peritoneal dialysis therapy

\begin{abstract}
Objective: To determine changes in lipid profile and safety of treatment with atorvastatin at high doses (greater than or equal to $40 \mathrm{mg} /$ day) in patients with stage 5 chronic kidney disease. Patients, materials and methods: Patients with stage 5 chronic kidney disease in peritoneal dialysis therapy and hypercholesterolemia refractory to therapy with lovastatin at a dose of $40 \mathrm{mg}$ daily and atorvastatin (less than $40 \mathrm{mg} /$ day) that were assigned to atorvastatin therapy at high doses.

Statistical analysis: A longitudinal study, retroprospective, correlational, single center with univariate and bivariate analysis.

Results: Twenty met the inclusion criteria. Time with high-dose atorvastatin averaged 27 months. The average dose used $60.4 \mathrm{mg} /$ day. The beneficial tendency to achieve therapeutic goals with time-varying was achieved in $65 \%$ of patients for total cholesterol, $50 \%$ LDL cholesterol, $40 \%$ non-HDL cholesterol and $45 \%$ for triglycerides. For variables LDL cholesterol and HDL cholesterol a statistically significant $(p<0.05)$ difference was found between the initial and final values. Twenty six complications were documented, none of them considered adverse events related to the administration of high-dose atorvastatin.

Conclusions: In patients with stage 5 chronic kidney disease over 50 years, peritoneal dialysis therapy and hypercholesterolemia, high-dose atorvastatin achieved LDL levels get goals in $50 \%$ of patients without significant adverse events.

(c) 2014 Sociedad Colombiana de Cardiología y Cirugía Cardiovascular. Published by Elsevier España, S.L.U. All rights reserved.
\end{abstract}

\section{Introducción}

La prevalencia e incidencia de la enfermedad renal crónica va en aumento progresivo, principalmente por incremento en las dos patologías que la generan: diabetes mellitus e hipertensión arterial crónica ${ }^{1,2}$. La mortalidad se genera en mayor forma por patologías cardiovasculares tipo cardiopatía isquémica, aconteciendo para el $40 \%$ de las muertes de pacientes en diálisis ${ }^{3-5}$; para esta última la dislipidemia constituye un factor de riesgo importante y modificable ${ }^{6,7}$.

En pacientes con enfermedad renal crónica estadio 5 (tasa de filtración glomerular - GFR su sigla en Inglés - menor a $15 \mathrm{~mL} / \mathrm{min}$ ) en terapia de diálisis, se ha observado mayor mortalidad por causas cardiovasculares (principalmente infarto de miocardio) en el programa de diálisis peritoneal que en el de hemodiálisis ${ }^{8}$.

La enfermedad renal crónica se caracteriza por la aparición progresiva de dislipidemia, producto del metabolismo anormal de las apolipoproteínas, presentándose reducción en las concentraciones de lipoproteínas que contienen apoA (HDL), pocos cambios en las concentraciones de lipoproteínas que contienen apoB ricas en colesterol (LDL e IDL) y aumento en las concentraciones de lipoproteínas intactas y parcialmente metabolizadas que contienen apoB ricas en triglicéridos (VLDL). Ello ocurre como resultado de disminución en su catabolismo (por reducción en la actividad de enzimas lipolíticas) y depuración por reducción en la captación mediada por receptores de lipoproteínas 9 .

La hemodiálisis puede atenuar moderadamente la dislipidemia renal, cuya principal característica es la elevación en triglicéridos, pero contrariamente la diálisis peritoneal se asocia con mayor agravamiento de la misma con incremento de las lipoproteínas que contienen apoB ricas en colesterol.

El tratamiento de la dislipidemia, principalmente aquella con un perfil aterogénico franco, como la que presentan los pacientes en diálisis peritoneal, debe convertirse en una alternativa importante que pueda modificar los factores de riesgo cardiovascular. Las estatinas representan el grupo terapéutico más importante para intervenir el perfil aterogénico, y la atorvastatina por su perfil de seguridad en teoría representaría el medicamento de elección en pacientes con enfermedad renal crónica ${ }^{10}$. Este trabajo de investigación buscó aclarar si la atorvastatina en dosis más altas que las convencionales puede mejorar de manera significativa el perfil lipídico de los pacientes con enfermedad renal crónica estadio 5 en diálisis peritoneal, sin generar efectos colaterales importantes.

\section{Pacientes, materiales y métodos}

Para la evaluación inicial se seleccionaron pacientes con enfermedad renal crónica estadio 5, mayores de 18 años, que se encontraran en terapia de diálisis peritoneal, con hipercolesterolemia refractaria a la terapia con lovastatina $40 \mathrm{mg} /$ día o en dosis bajas de atorvastatina (menores a $40 \mathrm{mg} /$ día) y con persistencia de colesterol LDL fuera de metas $(>100 \mathrm{mg} / \mathrm{dL}$ ) a pesar de la terapia, y en quienes se había formulado previamente una dosis alta de atorvastatina mayor o igual a $40 \mathrm{mg} /$ día y asistían a control mensual por Nefrología. En ellos fueron criterios de exclusión para participar en el estudio: historia de reacción de hipersensibilidad a atorvastatina u otras estatinas, hepatopatía conocida, AST 
y/o ALT o CK total $\geq 3$ veces el límite superior de normalidad, antecedente de rabdomiólisis o miopatía por estatinas, historia de alcoholismo o consumo de sustancias psicoactivas, neoplasia activa en manejo y terapia con atorvastatina en dosis altas por un periodo de tiempo menor a 12 meses. En este grupo de pacientes se evaluaron, desde el inicio de la atorvastatina, perfil lipídico, pruebas de funcion hepática, glucemia en ayunas, HbA1c, albúmina sérica, entre otras variables, de manera periódica cada 4 meses según los controles habituales establecidos por Nefrología, siendo determinados por los métodos de laboratorio rutinarios.

Se consideró meta terapéutica valores de colesterol total, LDL, no-HDL y triglicéridos menores a 200, 100, 130 y $150 \mathrm{mg} / \mathrm{dL}$, respectivamente.

El trabajo de investigación fue aprobado por docentes del Departamento Clínico de la Facultad de Ciencias para la Salud y el Comité de Bioética de la Facultad de Ciencias para la Salud de la Universidad de Caldas.

Todos los pacientes firmaron consentimiento pleno e informado en el cual autorizaron revisar su historia clínica y la toma de los datos necesarios para obtener los resultados del estudio, a pesar de que el estudio era solo observacional, descriptivo, no experimental, longitudinal, retroprospectivo, correlacional, dado que el uso de atorvastatina en dosis altas (hasta $80 \mathrm{mg} /$ día) está aprobado en el mundo para pacientes con enfermedad renal crónica estadio $5^{3}$, considerándose, por tanto, como un estudio con riesgo mínimo (inherente a la investigación).

La recolección de la información se hizo mediante una base de datos diseñada en Excel la cual fue diligenciada por los investigadores del proyecto y después fue leída y procesada en el programa SPSS versión 17.0.

Para el análisis estadístico las variables cuantitativas se definieron con medias y desviación estándar. Las variables cualitativas se describieron con proporciones y frecuencias absolutas. A las variables se les aplicó la prueba de normalidad de Shapiro-Wilk; cuando la distribución fue normal se aplicó la prueba de $t$ de Student para muestras relacionadas y cuando la distribución no lo fue, la prueba no paramétrica de Wilcoxon. Para estimar las diferencias entre las categorías de las variables cualitativas se usó la prueba de chi cuadrado $\left(\chi^{2}\right)$ y se utilizó como nivel de significación estadística $p \leq 0,05$. Los resultados se presentaron en tablas uni- y bivariadas, y en gráficos de acuerdo con las variables estudiadas.

\section{Resultados}

Se evaluaron 138 historias clínicas de pacientes en programa de diálisis peritoneal; 28 de ellos cumplieron con la mayoría de los criterios de inclusión, pero 7 llevaban un periodo de terapia con atorvastatina en dosis altas menor a 12 meses, y un paciente fue excluido por mieloma múltiple.

En total se incluyeron 20 pacientes en el estudio. Las características generales se muestran en la tabla 1.

En el momento de inicio de la administración de atorvastatina 19 pacientes $(95 \%)$ presentaron cifras de colesterol total mayores de $200 \mathrm{mg} / \mathrm{dL}$ y $20(100 \%)$ cifras de colesterol LDL mayores de 100 . Además, 5 pacientes $(25 \%)$ tenían colesterol HDL menor de $40 \mathrm{mg} / \mathrm{dL}$, 20 pacientes tenían colesterol
Tabla 1 Características de base de los pacientes

\begin{tabular}{|c|c|}
\hline Característica & $\begin{array}{l}\text { Participantes } \\
(\mathrm{n}=20)\end{array}$ \\
\hline 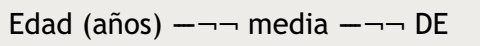 & $58,7 \pm 11,49$ \\
\hline Sexo femenino - no. (\%) & $15(75)$ \\
\hline Sexo masculino - no. (\%) & $5(25)$ \\
\hline Raza mestiza - no. (\%) & $20(100)$ \\
\hline $\begin{array}{l}\text { Tasa de filtración glomerular } \\
(\mathrm{mL} / \mathrm{min})-\text { media- } \neg \neg \mathrm{DE}\end{array}$ & $10,03 \pm 3,19$ \\
\hline \multicolumn{2}{|l|}{$\begin{array}{l}\text { Tipo de diálisis peritoneal - no. } \\
\text { (\%) }\end{array}$} \\
\hline CAPD & $13(65)$ \\
\hline APD & $7(35)$ \\
\hline $\begin{array}{l}\text { Tiempo en diálisis (meses) - } \neg \\
\text { media - DE }\end{array}$ & $72 \pm 45,8$ \\
\hline \multicolumn{2}{|l|}{$\begin{array}{l}\text { Etiología de la enfermedad renal } \\
\text { crónica-G5 - no. (\%) }\end{array}$} \\
\hline Nefropatía hipertensiva & $8(40)$ \\
\hline Nefropatía diabética & $7(35)$ \\
\hline Desconocida & $2(10)$ \\
\hline $\begin{array}{l}\text { Nefritis intersticial crónica } \\
\text { secundaria a AINE }\end{array}$ & $1(5)$ \\
\hline $\begin{array}{l}\text { Glomeruloesclerosis focal y } \\
\text { segmentaria primaria }\end{array}$ & $1(5)$ \\
\hline Nefropatía obstructiva & $1(5)$ \\
\hline \multicolumn{2}{|l|}{$\begin{array}{l}\text { Comorbilidades al inicio de } \\
\text { atorvastatina - no. (\%) }\end{array}$} \\
\hline Hipertensión arterial crónica & $20(100)$ \\
\hline Diabetes mellitus tipo 2 & $8(40)$ \\
\hline $\begin{array}{l}\text { Hipotiroidismo tratado } \\
\text { adecuadamente }\end{array}$ & $7(35)$ \\
\hline Hiperuricemia & $5(25)$ \\
\hline Enfermedad coronaria & $5(25)$ \\
\hline Enfermedad arterial periférica & $3(15)$ \\
\hline Enfermedad cerebrovascular & $2(10)$ \\
\hline $\begin{array}{l}\text { Colesterol total de base }(\mathrm{mg} / \mathrm{dL}) \\
\quad-\text { media } \triangle \neg \neg \mathrm{DE}\end{array}$ & $241 \pm 29,8$ \\
\hline $\begin{array}{l}\text { Colesterol LDL de base }(\mathrm{mg} / \mathrm{dL})- \\
\text { media } \neg \neg \neg \mathrm{DE}\end{array}$ & $153 \pm 25,2$ \\
\hline $\begin{array}{l}\text { Colesterol HDL de base }(\mathrm{mg} / \mathrm{dL})- \\
\text { media } \neg \neg \neg \mathrm{DE}\end{array}$ & $48,1 \pm 9,2$ \\
\hline $\begin{array}{l}\text { Colesterol no-HDL de base } \\
(\mathrm{mg} / \mathrm{dL})-\text { media }-\neg \neg \mathrm{DE}\end{array}$ & $192,8 \pm 29,8$ \\
\hline $\begin{array}{l}\text { Triglicéridos de base }(\mathrm{mg} / \mathrm{dL})- \\
\text { media } \neg \neg \neg \mathrm{DE}\end{array}$ & $197,6 \pm 71,7$ \\
\hline $\begin{array}{l}\text { PTHi }(\mathrm{pg} / \mathrm{mL}) \text { de base }-\neg \neg \text { media } \\
\quad-\neg \neg \mathrm{DE}\end{array}$ & $222 \pm 84,4$ \\
\hline $\begin{array}{l}\text { Calcio sérico total }(\mathrm{mg} / \mathrm{dL}) \text { de } \\
\text { base }-\neg \neg \text { media }-\neg \neg D E\end{array}$ & $9,2 \pm 0,74$ \\
\hline $\begin{array}{l}\text { Fosforo sérico }(\mathrm{mg} / \mathrm{dL}) \text { de base } \\
\qquad \neg \neg \text { media } \neg \neg \neg D E\end{array}$ & $4,9 \pm 1,15$ \\
\hline $\begin{array}{l}\text { Hemoglobina }(\mathrm{g} / \mathrm{dL}) \text { de base } \\
\quad-\neg \neg \text { media }-\neg \neg \mathrm{DE}\end{array}$ & $12,2 \pm 1,4$ \\
\hline $\begin{array}{l}\text { Albúmina }(\mathrm{g} / \mathrm{dL}) \text { de base } \\
\quad-\neg \neg \text { media }-\neg \neg D E\end{array}$ & $3,6 \pm 0,47$ \\
\hline $\begin{array}{l}\text { Glucemia }(\mathrm{mg} / \mathrm{dL}) \text { de base } \\
\quad \_\neg \neg \text { media }-\neg \neg D E\end{array}$ & $119 \pm 54,8$ \\
\hline $\begin{array}{l}\text { Fosfatasa alcalina }(\mathrm{U} / \mathrm{l}) \text { de base } \\
-\neg \neg \neg \text { media }-\neg \neg \mathrm{DE}\end{array}$ & $173,8 \pm 111,5$ \\
\hline
\end{tabular}




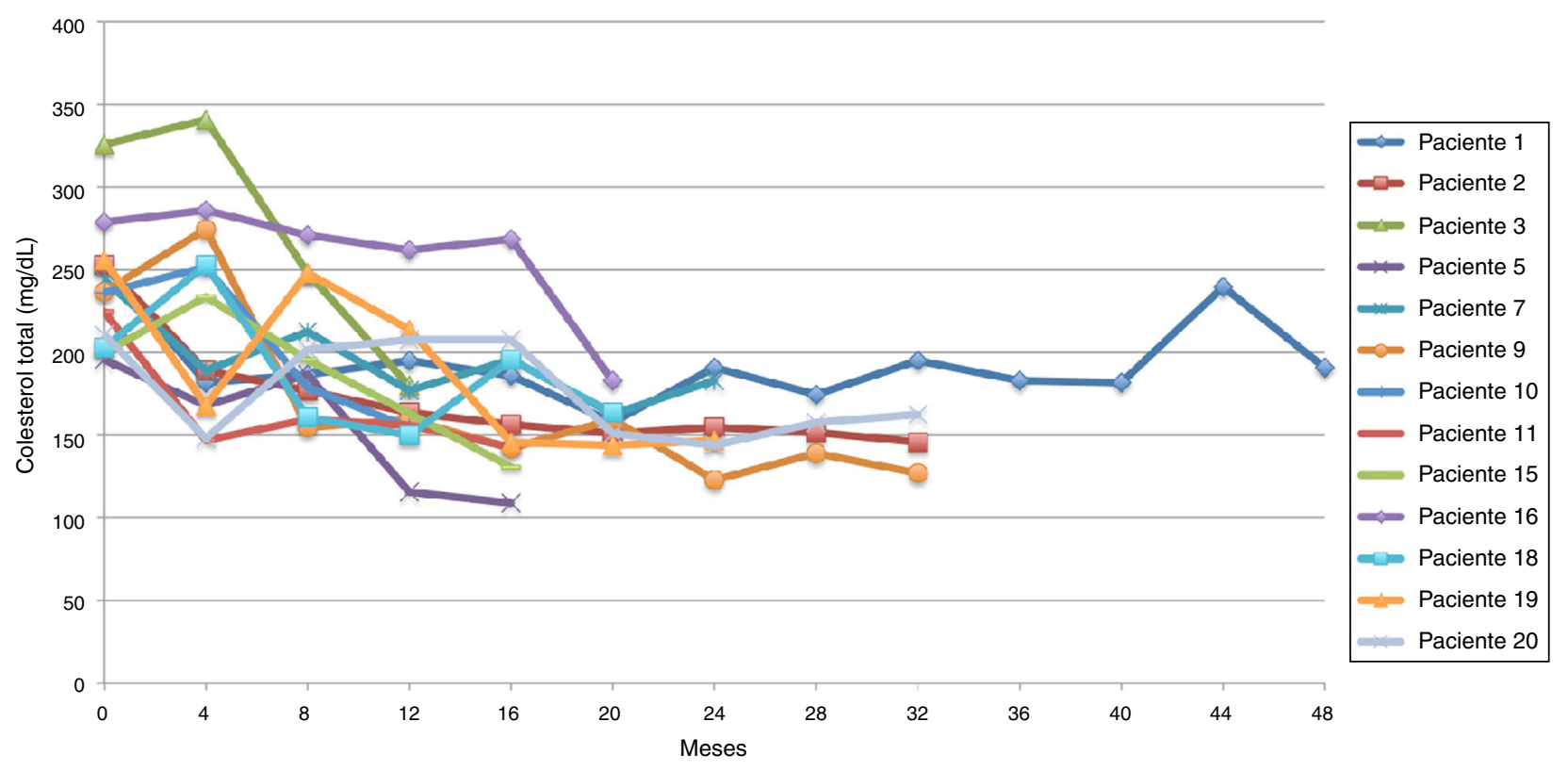

Figura 1 Tendencia 1 en colesterol total.

no-HDL mayor de 130 mg/dL y 14 (70\%) cifras de triglicéridos mayores de $150 \mathrm{mg} / \mathrm{dL}$.

En la medicación concomitante relevante para la atorvastatina los medicamentos más comunes fueron calcioantagonistas dihidropiridínicos en 16 pacientes (80\%), betabloqueadores en $13(65 \%)$ y calcioantagonistas no dihidropiridínicos en 3 (15\%).

El tiempo en terapia con atorvastatina fue en promedio de 27 meses (12-51; DE 10,53). La dosis promedio de atorvastatina utilizada a los $8,12,24,36$ y 44 meses fue de: 68 , 64, 64, 66 y $40 \mathrm{mg} /$ día respectivamente.

Las variables: colesterol total, LDL, HDL, no-HDL y triglicéridos se evaluaron cada 4 meses desde el momento 0 (inicio de la terapia) hasta que finalizó el seguimiento. Se establecieron los cambios en las variables lipídicas entre 0-12 meses, 0-24 meses y 0-36 meses. Además, según el comportamiento de las variables en el tiempo se establecieron tendencias.

El promedio de cambio de colesterol total entre $0-12$ meses fue de $46,5 \mathrm{mg} / \mathrm{dL}$ ( $\pm 38,5 \mathrm{mg} / \mathrm{dL})$, entre $0-24$ meses $69,2 \mathrm{mg} / \mathrm{dL}( \pm 34,2 \mathrm{mg} / \mathrm{dL})$ y entre $0-36$ meses $60,3 \mathrm{mg} / \mathrm{dL}$ $( \pm 15 \mathrm{mg} / \mathrm{dL})$. Se establecieron dos tendencias: la tendencia uno finalizando seguimiento con colesterol total en metas (menor de $200 \mathrm{mg} / \mathrm{dL}$ ) se encontró en 13 pacientes (65\%), y la tendencia 2 con colesterol total fuera de metas, en 7 (figs. 1 y 2 ).

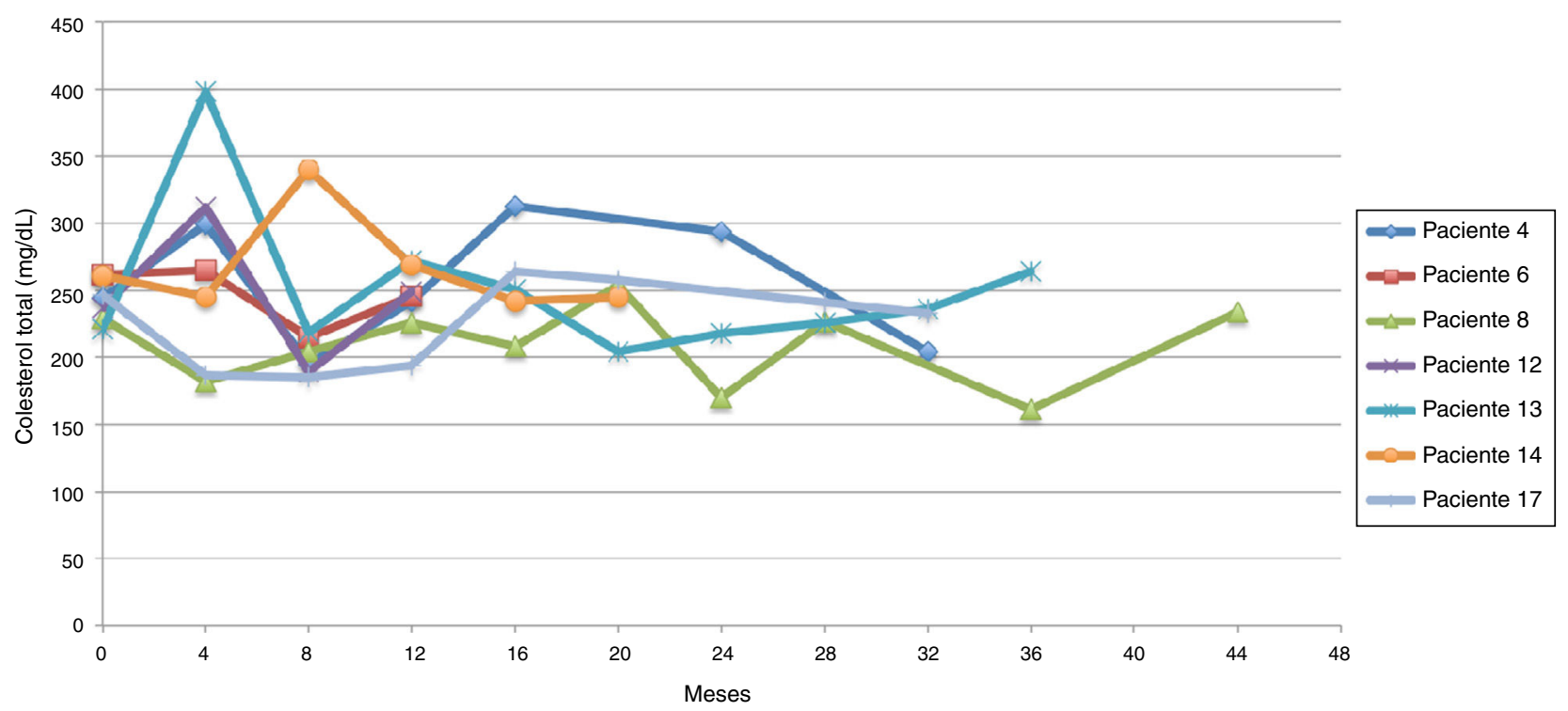

Figura 2 Tendencia 2 en colesterol total. 


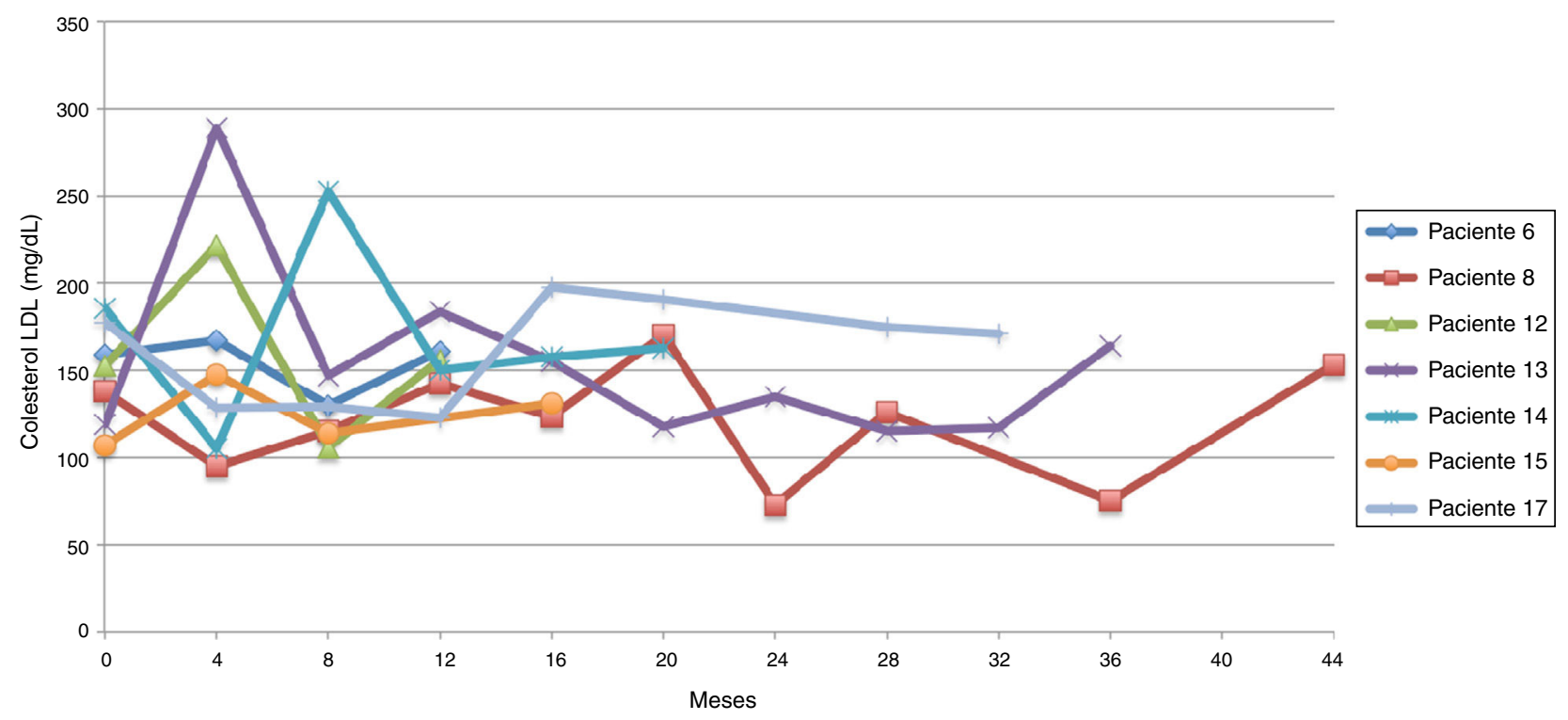

Figura 3 Tendencia 1 en colesterol LDL.

El promedio de cambio de colesterol LDL entre 0-12 meses fue de $34,5 \mathrm{mg} / \mathrm{dL}$ ( $\pm 42,6 \mathrm{mg} / \mathrm{dL}$ ), entre 0 - 24 meses $42,3 \mathrm{mg} / \mathrm{dL}( \pm 74,2 \mathrm{mg} / \mathrm{dL})$ y entre $0-36$ meses $27,6 \mathrm{mg} / \mathrm{dL}$ ( $\pm 62,9 \mathrm{mg} / \mathrm{dL})$.

Al evaluar el colesterol LDL al final del seguimiento se encontraron 10 pacientes (50\%) en metas ( $\mathrm{LDL}<100 \mathrm{mg} / \mathrm{dL})$; de estos, 5 (50\%) con LDL menor a $70 \mathrm{mg} / \mathrm{dL}$. En los pacientes con colesterol LDL fuera de metas se encontraron $4(20 \%)$ con cifras de colesterol LDL entre $100-150 \mathrm{mg} / \mathrm{dL}$ y $6(30 \%)$ con cifras de colesterol LDL mayores a $150 \mathrm{mg} / \mathrm{dL}$.

Siguiendo la tendencia uno (colesterol LDL en ascenso, fuera de metas) se encontraron 7 pacientes (35\%), bajo la tendencia 2 (colesterol LDL en descenso o cerca de metas) 3 pacientes (15\%) y bajo la tendencia 3 (colesterol LDL en metas) 10 pacientes (50\%) (figs. 3-5). La reducción del colesterol LDL en la tendencia 3 fue en promedio $79,2 \mathrm{mg} / \mathrm{dL}$ (37-133 mg/dL).

El promedio de cambio de colesterol no-HDL entre $0-12$ meses fue de $44,8 \mathrm{mg} / \mathrm{dL}$ ( $\pm 48,3 \mathrm{mg} / \mathrm{dL}$ ), entre $0-24$ meses $51,4 \mathrm{mg} / \mathrm{dL}( \pm 60,2 \mathrm{mg} / \mathrm{dL})$ y entre $0-36$ meses $23,6 \mathrm{mg} / \mathrm{dL}$
( $\pm 80,3 \mathrm{mg} / \mathrm{dL}$ ). Al evaluar el colesterol no-HDL, al final del seguimiento se lograron metas (no-HDL $<130 \mathrm{mg} / \mathrm{dL}$ ) en 8 pacientes $(40 \%), 6$ casos $(30 \%)$ de colesterol no-HDL entre $130-160 \mathrm{mg} / \mathrm{dL}$ y 6 (30\%) colesterol no-HDL mayor a 160 $\mathrm{mg} / \mathrm{dL}$. Siguiendo la tendencia uno (colesterol no-HDL en metas) se encontraron 8 pacientes (40\%), bajo la tendencia 2 (colesterol no-HDL con tendencia a metas, pero fuera de metas) 6 pacientes (30\%), y siguiendo la tendencia 3 (colesterol no-HDL en ascenso fuera de metas), 6 pacientes (30\%). La reducción del colesterol no-HDL en la tendencia uno fue en promedio $78 \mathrm{mg} / \mathrm{dL}(39-110 \mathrm{mg} / \mathrm{dL})$.

El promedio de cambio de colesterol $\mathrm{HDL}$ entre 0-12 meses fue de $2,1 \mathrm{mg} / \mathrm{dL}( \pm 10,7 \mathrm{mg} / \mathrm{dL})$, entre 0 24 meses $6,7 \mathrm{mg} / \mathrm{dL}( \pm 13,1 \mathrm{mg} / \mathrm{dL})$ y entre $0-36$ meses $7,8 \mathrm{mg} / \mathrm{dL}$ ( $\pm 16,6 \mathrm{mg} / \mathrm{dL})$. Al valorar el comportamiento del colesterol HDL al final del seguimiento se lograron metas ( $\mathrm{HDL} \geq 60 \mathrm{mg} / \mathrm{dL}$ ) solo en uno de los pacientes (5\%); 8 pacientes (40\%) tuvieron colesterol HDL entre 40 $59 \mathrm{mg} / \mathrm{dL}$ y los 11 restantes (55\%) colesterol HDL menor a $40 \mathrm{mg} / \mathrm{dL}$.

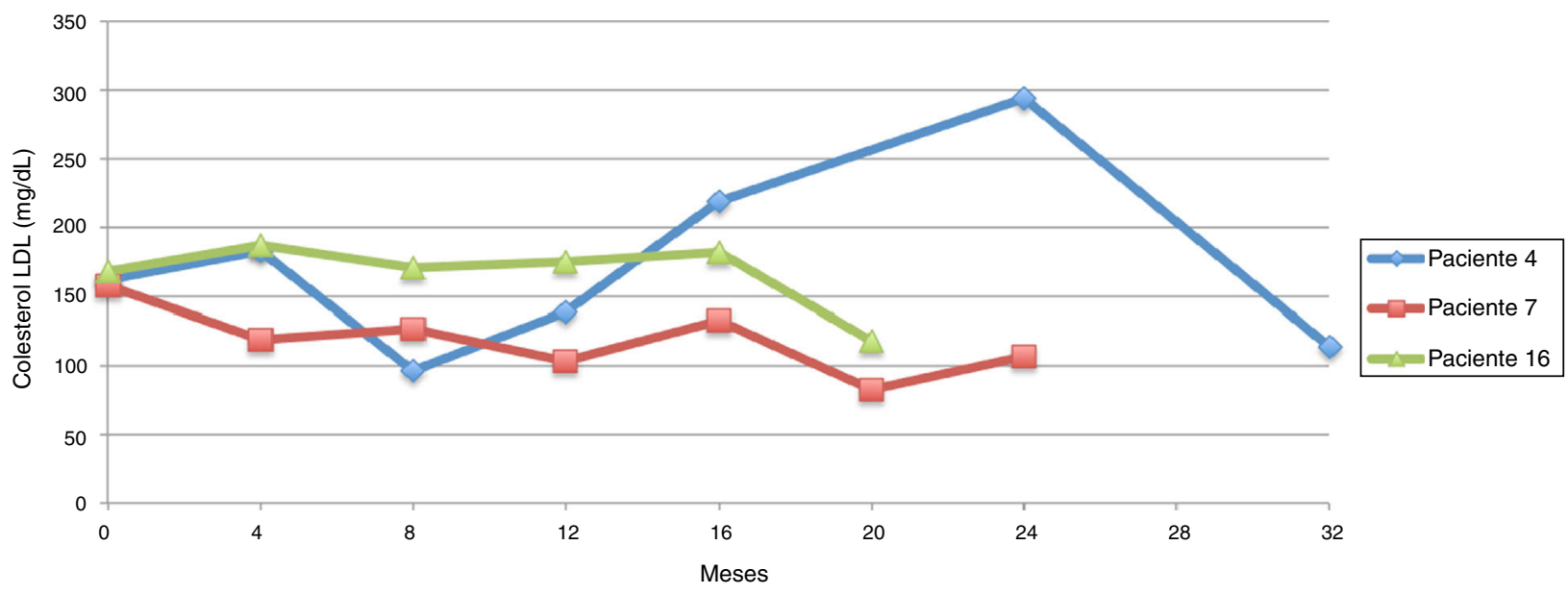

Figura 4 Tendencia 2 en colesterol LDL. 


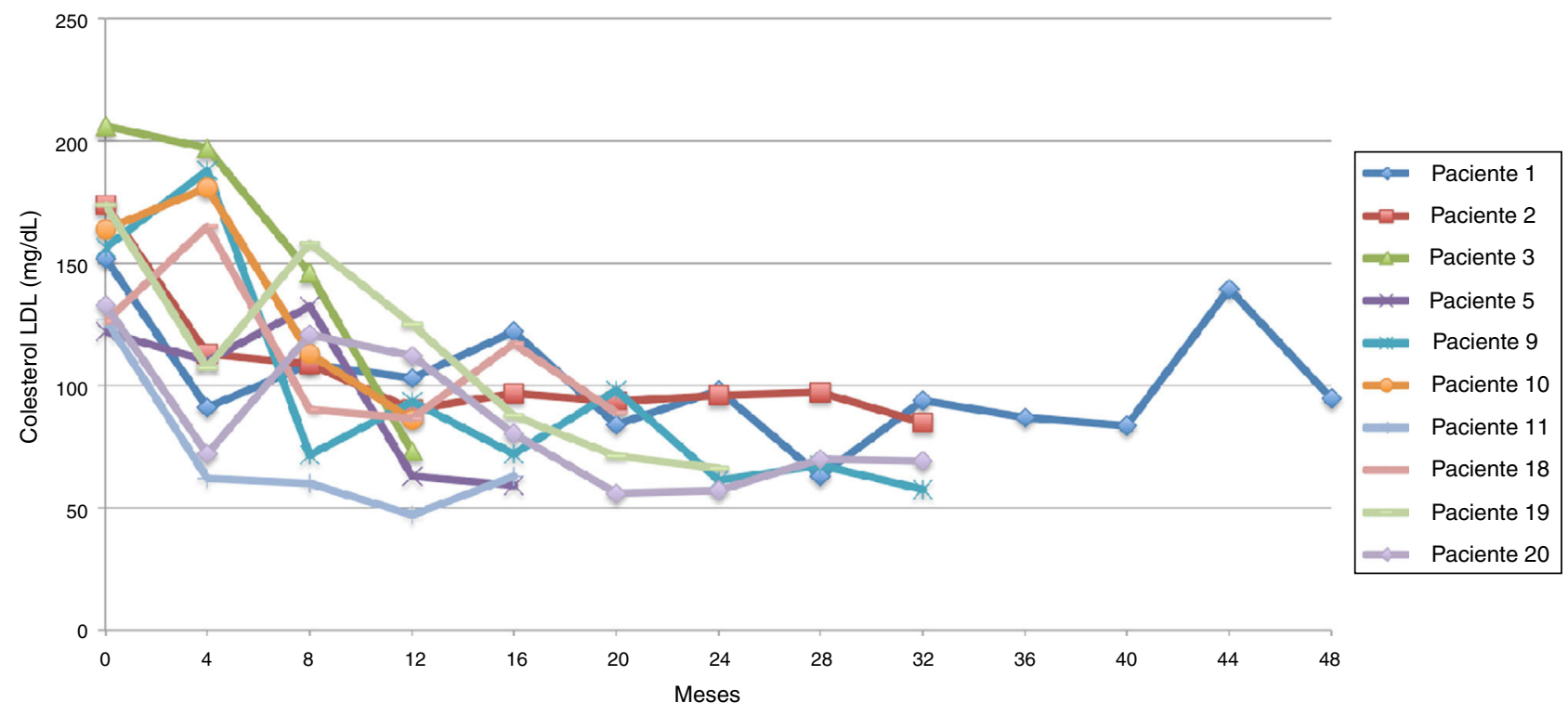

Figura 5 Tendencia 3 en colesterol LDL.

El promedio de cambio de triglicéridos entre 0-12 meses fue de $11,3 \mathrm{mg} / \mathrm{dL}( \pm 95,7 \mathrm{mg} / \mathrm{dL})$, entre $0-24$ meses 5,2 $\mathrm{mg} / \mathrm{dL}( \pm 102,1 \mathrm{mg} / \mathrm{dL})$ y entre $0-36$ meses $18,3 \mathrm{mg} / \mathrm{dL}$ $( \pm 91,9 \mathrm{mg} / \mathrm{dL})$. Cuando se evaluaron las cifras de triglicéridos al final del seguimiento se encontraron 9 pacientes (45\%) con triglicéridos menores a $150 \mathrm{mg} / \mathrm{dl}$, y los 11 restantes $(55 \%)$ con cifras de triglicéridos mayores o iguales a $150 \mathrm{mg} / \mathrm{dL}$. Ninguno presentó cifras de hipertrigliceridemia severa (triglicéridos $>500 \mathrm{mg} / \mathrm{dL}$ ).

Siguiendo la tendencia uno (triglicéridos $<150 \mathrm{mg} / \mathrm{dL}$ ) se encontraron 9 pacientes (45\%), y bajo la tendencia 2 (triglicéridos $\geq 150 \mathrm{mg} / \mathrm{dL}$ ) los 11 restantes (55\%) (figs. 6 y 7).

En el análisis bivariado a las variables colesterol total, LDL, HDL, no-HDL y triglicéridos de los 20 pacientes participantes en el estudio (en sus valores iniciales y finales) se aplicó la prueba de normalidad de Shapiro-Wilk por ser un número menor de 30 sujetos. A estas tres variables se les aplicó la prueba $\mathrm{t}$ de Student para muestras relacionadas; los resultados obtenidos en este análisis evidencian que no existe diferencia estadísticamente significativa entre valores iniciales y valores finales de colesterol no-HDL y triglicéridos $(p>0,05)$, pero sí hubo diferencia estadísticamente significativa entre valores iniciales y valores finales de colesterol total $(p<0,05)$.

A las variables colesterol LDL y colesterol HDL que no se distribuyeron normalmente en la prueba de Shapiro-Wilk, se les aplicó la prueba no paramétrica de Wilcoxon y se encontró diferencia estadísticamente significativa entre los valores iniciales y finales obtenidos en los 20 pacientes participantes en el estudio en estas dos variables $(p<0,05)$, (tablas 2-6).

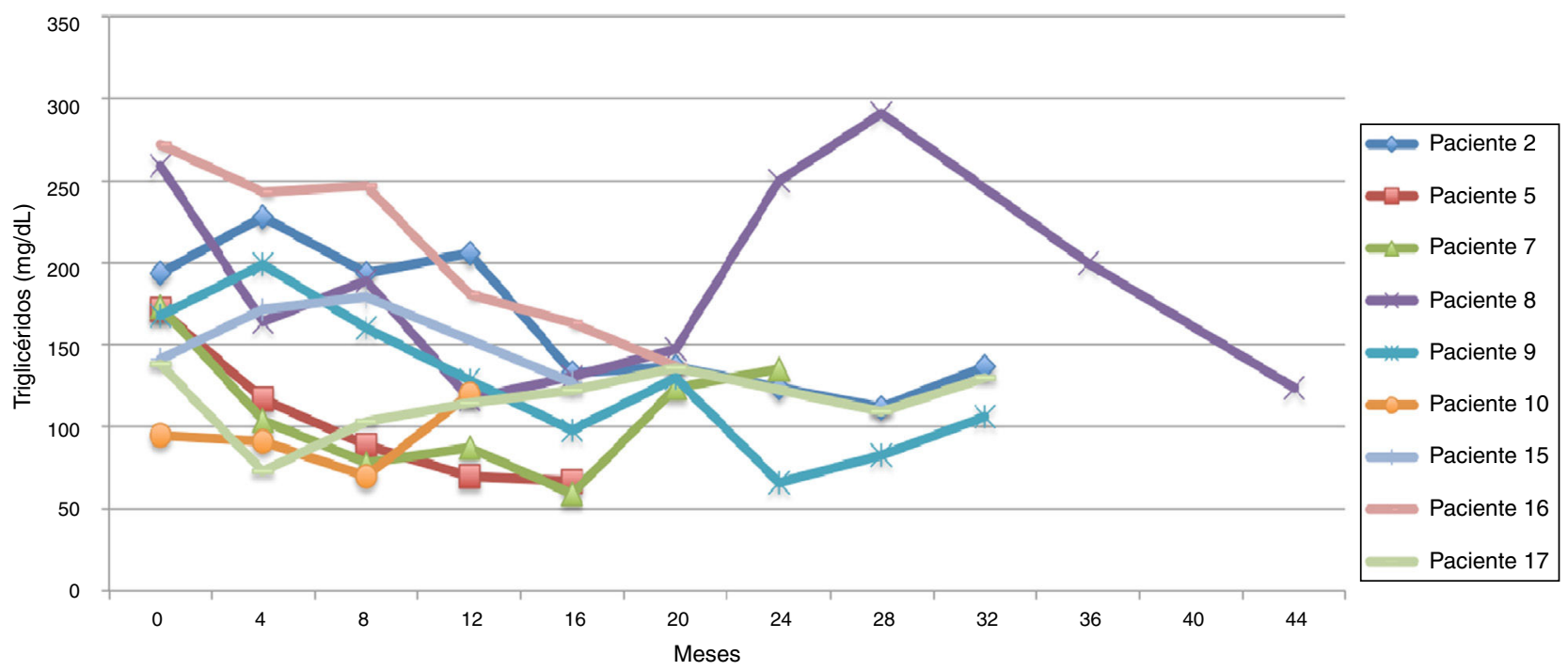

Figura 6 Tendencia 1 en triglicéridos. 


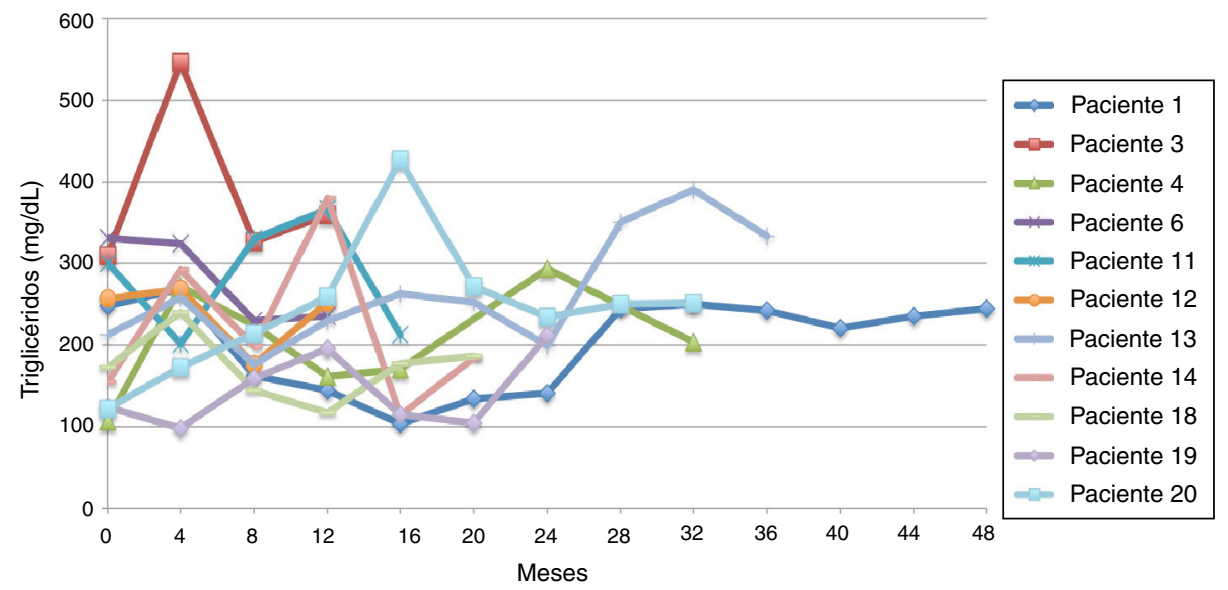

Figura 7 Tendencia 2 en triglicéridos.

Tabla 2 Prueba t de Student para muestras relacionadas, colesterol total

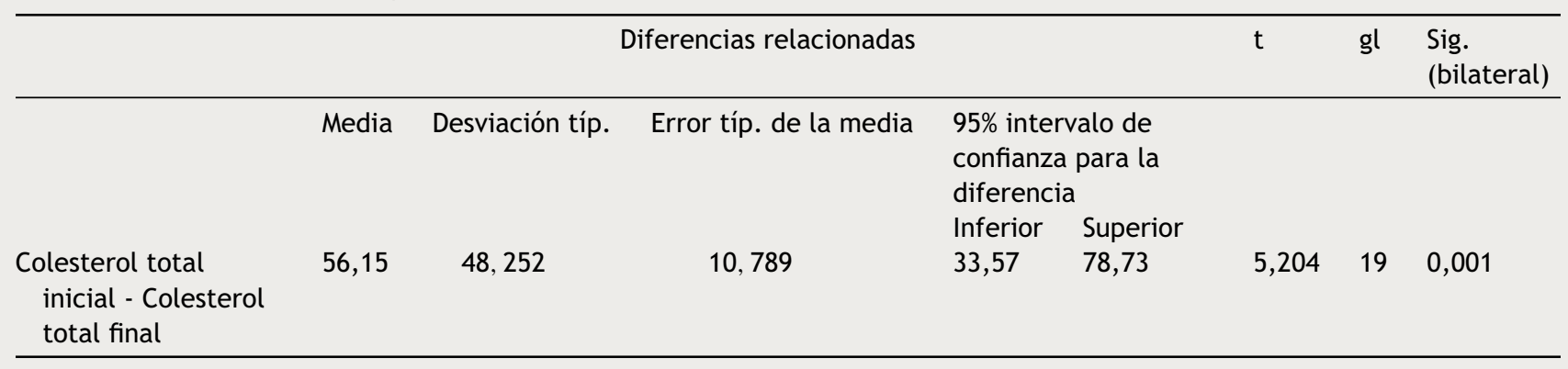

Tabla 3 Pruebas de normalidad, colesterol LDL

\begin{tabular}{llll}
\hline & \multicolumn{3}{c}{ Shapiro-Wilk } \\
\cline { 2 - 4 } & Estadístico & gl & Sig. \\
\hline Valor inicial LDL & 0,975 & 20 & 0,847 \\
Valor final LDL & 0,894 & 20 & 0,032 \\
\hline
\end{tabular}

Durante el tiempo de seguimiento se presentaron 4 desenlaces fatales. Todas las muertes se presentaron después de quince meses de seguimiento, 3 de los 4 pacientes recibían dosis de atorvastatina de $40 \mathrm{mg} /$ día y solo un paciente recibía $80 \mathrm{mg} /$ día. Hubo un caso de hemorragia subaracnoidea espontánea por rotura de aneurisma cerebral en mujer anticoagulada por accidente cerebrovascular cardioembólico previo.

Se detectaron 11 eventos cardiovasculares no fatales en 8 pacientes. En 3 pacientes enfermedad arterial oclusiva de miembros inferiores, 2 casos de infarto agudo de miocardio
Tabla 5 Pruebas de normalidad, colesterol HDL

\begin{tabular}{llll}
\hline & \multicolumn{3}{c}{ Shapiro-Wilk } \\
\cline { 2 - 4 } & Estadístico & $\mathrm{gl}$ & Sig. \\
\hline Valor inicial HDL & 0,954 & 20 & 0,426 \\
Valor final HDL & 0,900 & 20 & 0,041 \\
\hline
\end{tabular}

sin elevación del ST, 4 casos de accidente cerebrovascular y 2 ataques isquémicos transitorios.

En relación con la seguridad de la administración de la atorvastatina, no se encontraron en el seguimiento modificaciones importantes relacionadas con la medicación, en los valores de las enzimas hepáticas ni fosfatasa alcalina (fig. 8). Seis pacientes (30\%) presentaron síntomas musculares, todos con niveles de CPK normales; ninguno requirió suspensión de atorvastatina para resolución de los síntomas.

Tabla 6 Prueba no paramétrica de Wilcoxon, colesterol HDL

LDL

Valor final LDL - valor inicial LDL

\begin{tabular}{lc}
\hline$Z$ & $-3,136(\mathrm{a})$ \\
Sig. asintót. (bilateral) & 0,002 \\
\hline
\end{tabular}

\begin{tabular}{lr}
\hline & Valor final HDL - valor inicial HDL \\
\hline$Z$ & $-2,334(a)$ \\
Sig. asintót. (bilateral) & 0,020 \\
\hline
\end{tabular}




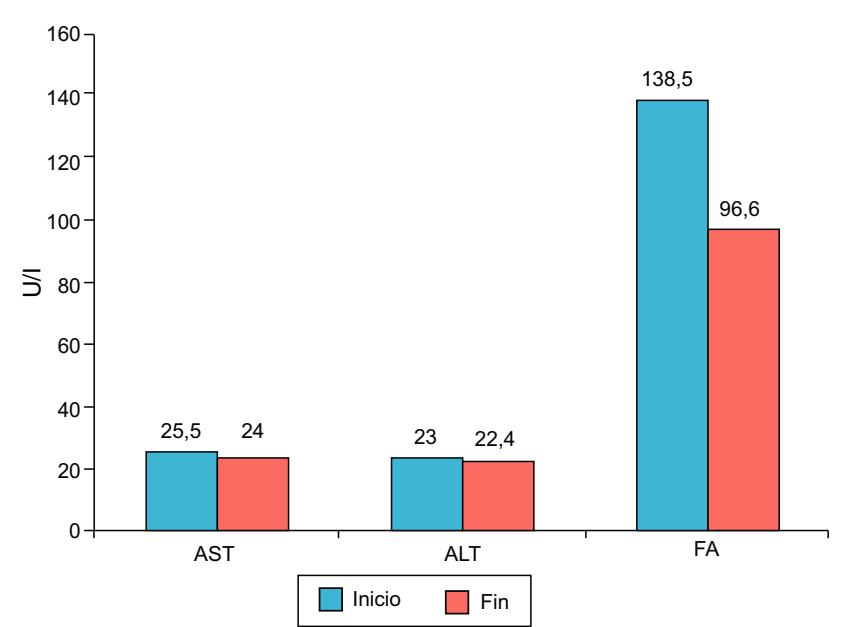

Figura 8 Evolución enzimas hepáticas (AST y ALT) y fosfatasa alcalina (FA).

\section{Discusión}

La dislipidemia renal tiene características diferentes en pacientes con enfermedad renal crónica estadio 5 en terapia dialítica. Los pacientes en hemodiálisis presentan principalmente hipertrigliceridemia con incrementos en las apoB ricas en triglicéridos, VLDL e IDL, niveles bajos de apoAı y apoAı, con disminución en los niveles de HDL, moderada elevación de apoB y apoE, aumento significativo de apoC-III, con pocos cambios en los niveles de lipoproteínas ricas en colesterol (LDL) $)^{11}$.

En pacientes en diálisis peritoneal es más común la dislipidemia, y además de la hipertrigliceridemia se detecta elevación en los valores de lipoproteínas que contienen apoB ricas en colesterol, lo cual se refleja en valores altos de ambas IDL y LDL, con mayor incremento en los niveles de apoB, apoC-III y apoE que en los pacientes en hemodiálisis, generándose en ellos un perfil más aterogénico ${ }^{12-15}$.

La diálisis peritoneal es un tratamiento que aporta gran cantidad de glucosa, parte de la cual se absorbe, aportando un sustrato, que puede aumentar la síntesis de lipoproteínas, agravando la dislipidemia ${ }^{14}$. A favor de ello está la utilización nocturna de solución de diálisis con icodextrin (libre de glucosa) que puede traducirse en una reducción moderada en el colesterol plasmático ${ }^{16}$. También es posible que la pérdida de proteínas en el líquido de diálisis peritoneal, incluidas albúmina, apolipoproteínas y $\mathrm{HDL}$, pueda disparar mecanismos semejantes a los que ocurren en el síndrome nefrótico con incremento en lipoproteínas ricas en colesterol ${ }^{17}$.

La mayor mortalidad por causa cardiovascular, principalmente por infarto de miocardio, más vista en pacientes en diálisis peritoneal que en hemodiálisis, podría obedecer al perfil más aterogénico que ostentan ${ }^{8,18-20}$.

Existe controversia en relación con el tratamiento de la dislipidemia en pacientes con enfermedad renal crónica estadio 5.

Las guías de 2013 del American College of Cardiology/American Heart Association para el tratamiento del colesterol sanguíneo y la reducción del riesgo cardiovascular aterosclerótico en adultos, concluyen que las modificaciones en el estilo de vida son un componente crítico para reducir el riesgo por enfermedad cardiovascular aterosclerótica (ECVAS). Indican, además, que se encontró evidencia extensa y contundente que avala el uso de estatinas para la prevención de ECVAS en individuos con alto riesgo primario, y en prevención secundaria, pero sin falla cardíaca NYHA II-IV, ni en aquellos que se encuentren en hemodiálisis. Con los estudios analizados se concluyó que no había información suficiente con base en la cual se estuviera a favor o en contra del uso de estatinas; por tanto no se generaron recomendaciones con relación al inicio o la continuación de la terapia con estatinas en esos dos grupos. De igual forma, no se hallaron comentarios en cuanto a intervenciones en pacientes en terapia de diálisis peritoneal. Se resalta que la terapia con estatinas de moderada a alta intensidad para reducir el valor de colesterol LDL entre un 30 a 50\%, o más del $50 \%$ respectivamente, es un factor crítico para reducir la ECVAS, en forma tal que a mayor dosis de estatinas más beneficios en reducción del riesgo, al igual que a mayor presencia de factores de riesgo para ECVAS, mayor beneficio terapéutico de las estatinas sobre un periodo de 5 a 10 años. Otro aspecto destacable de las guías es la identificación de pacientes diabéticos tipo 1 o 2 entre 40 a 75 años y con colesterol LDL de 70 a $189 \mathrm{mg} / \mathrm{dL}$, o con un riesgo de ECVAS mayor al 7,5\% entre los 4 grupos de mayor beneficio en terapia con estatinas, dato relevante si se tiene en cuenta que un $30 \mathrm{a}$ $40 \%$ de los pacientes con enfermedad renal crónica en terapia dialítica son diabéticos. En relación con la terapia con estatinas de alta intensidad, se recomiendan, dado su perfil de seguridad, atorvastatina 40 a $80 \mathrm{mg}$ al día o rosuvastatina 20 a $40 \mathrm{mg} /$ día.

Concluye la guía que en individuos con enfermedad renal crónica en terapia de hemodiálisis y dislipidemia el beneficio potencial de reducir el riesgo de ECVAS, efectos adversos, interacción de medicamentos, cuidados y contraindicaciones, y elección de la dosis de estatinas son factores que orientan la decisión del clínico sobre el inicio y la continuación de la terapia con dichos medicamentos ${ }^{21}$.

Las Guías de la Sociedad Cardiovascular Canadiense para el diagnóstico y tratamiento de la dislipidemia y para la prevención de la enfermedad cardiovascular en adultos, actualizadas en 2012, y publicadas en $2013^{22}$, consideran que todo paciente adulto, independientemente de su edad, con la presencia del factor de riesgo de enfermedad renal crónica debe ser evaluado para dislipidemia. La aplicación del score de riesgo Framingham en dichos pacientes, permite estimar el riesgo a 10 años de desarrollar eventos cardiovasculares totales. Aquellos con enfermedad renal crónica y tasa de filtración glomerular menor a $45 \mathrm{~mL} / \mathrm{min} / 1,73 \mathrm{~m}^{2} \mathrm{se}$ consideran de alto riesgo para mayores eventos cardiovasculares, lo cual sugiere que este grupo se beneficia con la utilización de estatinas, recomendándose la meta terapéutica de un valor de LDL colesterol menor a 2,0 mmol/L (80 $\mathrm{mg} / \mathrm{dL}$ ), o una reducción mayor del $50 \%$ en relación con su valor inicial.

Las guías KDIGO de 2013, para el manejo de lípidos en enfermedad renal crónica ${ }^{23}$, establecen que a todo adulto con diagnóstico reciente de enfermedad renal crónica, incluidos aquellos en diálisis crónica, se le debe practicar perfil lipídico, aunque indican que no se requiere seguimiento del mismo. Para adultos mayores de 50 años, con enfermedad renal crónica (TFG menor a $60 \mathrm{~mL} / \mathrm{min}$ ) estadio 
G3 a G5 no tratados por diálisis crónica, con dislipidemia y riesgo cardiovascular, se recomienda el tratamiento con estatinas o la asociación ezetimibe/estatina. No obstante, en pacientes con enfermedad renal crónica dependientes de diálisis (no indican la variedad) sugieren no iniciar las estatinas o la combinación ezetimibe/estatina, aunque deberían continuarse en aquellos que recibían alguno de estos tipos de tratamientos previos al inicio de la diálisis. En cuanto al seguimiento y la dosificación de las estatinas de manera escalonada para obtener un blanco terapéutico (LDL menor a $100 \mathrm{mg} / \mathrm{dL}$ ), consideran que no hay suficiente evidencia para recomendar este proceso puesto que sus resultados poco darían lugar a cambios en el tratamiento; por tanto, sugieren la alternativa «dispare y olvide» («fire and forget»), aunque aclaran que el médico es quien debe definir la necesidad de practicar un seguimiento considerando que el mismo mejora la adherencia al tratamiento y otros procesos de atención. Es probable que los pacientes con muy altos valores de colesterol LDL en terapia de diálisis, tengan mayores beneficios de la terapia con estatinas, lo cual puede llevar al médico y al paciente a optar por la terapia con estatinas a pesar de los resultados no concluyentes de las pruebas expuestas ${ }^{24}$.

Existen varios estudios que han analizado los diversos trabajos de investigación llevados a cabo interviniendo pacientes con enfermedad renal crónica estadio 5 en terapia dialítica. Recientemente en Cochrane se revisaron 25 estudios al respecto, en los que concluyen que las estatinas tienen poco o ningún efecto benéfico en la mortalidad o en eventos cardiovasculares a pesar de lograr reducción en los niveles del colesterol sérico. Sin embargo, aclaran que existe poca información para pacientes tratados en diálisis peritoneal, hecho que sugiere la necesidad de más investigaciones en este grupo de pacientes ${ }^{25}$.

Tres metaanálisis recientes analizan los resultados de la terapia con estatinas en pacientes con enfermedad renal crónica, y generan más controversia al respecto. En el primero de ellos, el grupo de Upadhyay et al. ${ }^{26}$ concluyó que la terapia para disminuir lípidos decrece el riesgo para mortalidad cardiaca, eventos cardiovasculares e infarto de miocardio, aunque el beneficio de reducción de mortalidad para todas las causas solo ocurrió en pacientes que no se encontraban en terapia dialítica. En el segundo, de Palmer et al. ${ }^{27}$ las estatinas solo redujeron la mortalidad de todas las causas, mortalidad cardiovascular y eventos cardiovasculares en pacientes con enfermedad renal crónica que no estaban en terapia dialítica.

El tercero, último y más reciente, de Hou et al. ${ }^{28}$ concluyó que la terapia con estatinas sí produce una reducción significativa de eventos cardiovasculares mayores, eventos coronarios, muerte cardiovascular y por todas las causas a través de todo el rango de función renal, incluidos los pacientes en diálisis.

Cabe resaltar que en la discusión de estos se anota que la población de pacientes en diálisis no puede mezclarse, y que no hay suficientes estudios de pacientes en diálisis peritoneal con un perfil lipídico más aterogénico para analizarlos de manera individual. Sin embargo un estudio reciente de Lee et al. ${ }^{29}$ evaluó 1.024 pacientes incidentes a programas de diálisis peritoneal en 7 centros en Korea; a 37,8\% (387 pacientes) se le prescribió una estatina, y fueron comparados con igual número de pacientes sin la prescripción y con la misma etiología de enfermedad renal crónica. Se practicó seguimiento durante un promedio de 2,7 años, detectándose que la probabilidad de sobrevida para usuarios de estatinas vs. no usuarios fue del 87 vs. $80 \%$, y 76 vs. $69 \%$ a 3 y 5 años, respectivamente.

Otros factores que también pueden influir la decisión de iniciar tratamiento con estatinas en pacientes con enfermedad renal crónica estadio 5 en terapia dialítica son: infarto de miocardio reciente, mayor expectativa de vida y comorbilidad más severa, esta última representada por la concomitancia de otros factores de riesgo cardiovascular como la diabetes mellitus.

A favor de la elección individualizada de iniciar terapia de acuerdo con la presencia de factores de riesgo cardiovasculares en pacientes con enfermedad renal crónica estadio 5, están los subanálisis del 4D y del AURORA. En el análisis post hoc $4 \mathrm{D}$ se pudo observar que la administración de atorvastatina en pacientes con enfermedad renal crónica y diabetes mellitus en terapia hemodialítica con un alto cuartil de elevación del colesterol LDL (mayor a $145 \mathrm{mg} / \mathrm{dL}$ ), sí redujo significativamente eventos cardiovasculares como muerte cardíaca, muerte cardíaca súbita, infarto de miocardio no fatal y todos los eventos cardíacos combinados ${ }^{24,30,31}$. En otro análisis post hoc del estudio AURORA, que incluyó diabéticos y no diabéticos en hemodiálisis, se optó por analizar solo el grupo de pacientes diabéticos tratados con rosuvastatina y se encontró que la tasa de eventos cardíacos fatales y no fatales se redujo en un $32 \%{ }^{32}$.

\section{Conclusiones}

En pacientes con enfermedad renal crónica estadio 5, mayores de 18 años, en terapia de diálisis peritoneal e hipercolesterolemia, la atorvastatina en dosis altas logra obtener niveles de LDL en metas en el $50 \%$ de los pacientes tratados, sin que su administración dé lugar a eventos adversos significativos.

Este trabajo no pretende controvertir las guías actuales, sino demostrar que la atorvastatina en dosis altas (terapia de alta intensidad) es bien tolerada por pacientes con enfermedad renal crónica en terapia de diálisis peritoneal, y que el médico puede tomar la determinación de usarla o no según su criterio, con base en el perfil de riesgo cardiovascular que porta su paciente. La dosis y preocupación por la aparición de efectos colaterales, no debe, por tanto, constituir una limitación para la prescripción de atorvastatina de alta intensidad.

\section{Conflicto de intereses}

Los autores declaran no tener ningún conflicto de intereses.

\section{Bibliografía}

1. Gilbertson DT, Liu J, Xue JL, Louis TA, Solid CA, Ebben JP, et al. Projecting the number of patients with end-stage renal disease in the United States to the year 2015. J Am Soc Nephrol. 2005; $16: 3736-41$.

2. Atkins RC, Zimmet P. Diabetic kidney disease: act now or pay later. Nephrol Dial Transplant. 2010;25:331-3.

3. Kidney Disease Outcome Quality Initiative (K/DOQI) Group. AT K/DOQI clinical practice guidelines for management of 
dyslipidemias in patients with kidney disease. Am J Kidney Dis. 2003;41(I-IV):S1-91.

4. Go AS, Chertow GM, Fan D, McCulloch CE, Hsu CY. Chronic kidney disease and the risks of death, cardiovascular events, and hospitalization. N Engl J Med. 2004;351:1296-305.

5. De Jager DJ, Grootendorst DC, Jager KJ, van Dijk PC, Tomas LM, Ansell D. Cardiovascular and noncardiovascular mortality among patients starting dialysis. JAMA. 2009;302:1782-9.

6. Kasiske BL. Hyperlipidemia in patients with chronic renal disease. Am J Kidney Dis. 1998;32 Suppl. 3:S142-56.

7. Foley RN, Parfrey PS, Sarnak MJ. Epidemiology of cardiovascular disease in chronic renal disease. J Am Soc Nephrol. 1998;9 Suppl. 12:S16-23.

8. Johnson DW, Dent H, Hawley CM, McDonald SP, Rosman JB, Brown FG, et al. Association of dialysis modality and cardiovascular mortality in incident dialysis patients. Clin J Am Soc Nephrol. 2009;4:1620-8.

9. Attman PO, Samuelsson O, Alaupovic P. Lipoprotein metabolism and renal failure. Am J Kidney Dis. 1993;21:573-91.

10. Newman C, Tsai J, Szarek M, Lio D, Gibson E. Comparative safety of atorvastatin $80 \mathrm{mg}$ versus $10 \mathrm{mg}$ derived from analysis of 49 completed trials in 14,236 patients. Am J Cardiology. 2006;97:61-7.

11. Attman PO, Samuelsson O, Johansson AC, Moberly JB, Alaupovic P. Dialysis modalities and dyslipidemia. Kidney Int. 2003;63:S110-2.

12. Attman PO, Samuelsson O, Moberly J, Johansson AC, Ljungman S, Weiss LG, et al. Apolipoprotein B-containing lipoproteins in renal failure: The relation to mode of dialysis. Kidney Int. 1999;55:1536-42.

13. Moberly JB, Attman PO, Samuelsson O, Johansson AC, KnightGibson C, Alaupovic P. Alterations in lipoprotein composition in peritoneal dialysis patients. Perit Dial Int. 2002;22:220-8.

14. Johansson AC, Samuelsson O, Attman PO, Haraldsson B, Moberly $\mathrm{J}$, Knight-Gibson C, et al. Dyslipidemia in peritoneal dialysisrelation to dialytic variables. Perit Dial Int. 2000;20:306-14.

15. Wheeler DC. Abnormalities of lipoprotein metabolism in CAPD patients. Kidney Int. 1996;50 Suppl. 56:S41-6.

16. Bredie SJ, Bosch FH, Demacker PN, Stalenhoef AF, van Leusen R. Effects of peritoneal dialysis with an overnight icodextrin dwell on parameters of glucose and lipid metabolism. Perit Dial Int. 2001;21:275-81.

17. Kagan A, Bar-Khayim Y, Schafer Z, Fainaru M. Kinetics of peritoneal protein loss during CAPD. II. Lipoprotein leakage and its impact on plasma lipid levels. Kidney Int. 1990;37:980-90.

18. Bloembergen WE, Port FK, Mauger EA, Wolfe RA. A comparison of cause of death between patients treated with hemodialysis and peritoneal dialysis. J Am Soc Nephrol. 1995;6:184-91.

19. Ganesh SK, Hulbert-Shearon T, Port FK, Eagle K, Stack AG. Mortality differences by dialysis modality among incident ESRD patients with and without coronary artery disease. J Am Soc Nephrol. 2003;14:415-24.
20. Stack AG, Molony DA, Rahman NS, Dosekun A, Murthy B. Impact of dialysis modality on survival of new ESRD patients with congestive heart failure in the United States. Kidney Int. 2003;64:1071-9.

21. Stone NJ, Robinson J, Lichtenstein AH, Bairey Merz CN, Blum $\mathrm{CB}$, Eckel RH, et al. ACC/AHA guideline on the treatment of blood cholesterol to reduce atherosclerotic cardiovascular risk in adults: a report of the American College of Cardiology/American Heart Association Task Force on Practice Guidelines. J Am Coll Cardiol. 2013:S0735-1097.

22. Anderson TJ, Grégoire J, Hegele RA, Couture P, Mancini GB, McPherson R, et al. 2012 Update of the Canadian Cardiovascular Society Guidelines for the diagnosis and treatment of dyslipidemia for the prevention of cardiovascular disease in the adult. Can J Cardiol. 2013;29:151-67.

23. Tonelli MA, Wanner C, Cass A, Kronenberg F, Garg AX, Parekh RS, et al. KDIGO Clinical Practice Guideline for lipid management in chronic kidney disease. Kidney Int. 2013;3 Suppl. 3:259-305.

24. Marz W, Genser B, Drechsler C, Krane V, Grammer TB, Ritz E, et al. Atorvastatin and low-density lipoprotein cholesterol in type 2 diabetes mellitus patients on hemodialysis. Clin J Am Soc Nephrol. 2011;6:1316-25.

25. Palmer SC, Navaneethan SD, Craig JC, Johnson DW, Perkovic V, Nigwekar SU, et al. HMG CoA reductase inhibitors (statins) for dialysis patients. Cochrane Database Syst Rev. 2013;11(9):CD004289.

26. Upadhyay A, Earley A, Lamont JL, Haynes S, Wanner C, Balk EM. Lipid-lowering therapy in persons with chronic kidney disease: a systematic review and meta-analysis. Ann Intern Med. 2012;157:251-62.

27. Palmer SC, Craig JC, Navaneethan SD, Tonelli M, Pellegrini F, Strippoli GF. Benefits and harms of statin therapy for persons with chronic kidney disease: a systematic review and metaanalysis. Ann Intern Med. 2012;157:263-75.

28. Hou WH, Jicheng LV, Perkovic V, Yang L, Zhao N, Jardine MJ, et al. Effect of statin therapy on cardiovascular and renal outcomes in patients with chronic kidney disease: a systematic review and meta-analysis. Eur Heart J. 2013;34:1807-17.

29. Lee JE, Oh KH, Choi KH, Kim SB, Do JY, Kim YL, et al. Statin therapy is associated with improved survival in incident peritoneal dialysis patients: propensity-matched comparison. Nephrol Dial Transplant. 2011;26:4090-4.

30. Wanner C, Krane V, Marz W. Atorvastatin in patients with type 2 diabetes mellitus undergoing hemodialysis. N Engl J Med. 2005;353:238-48.

31. Wanner C, Schmidt KR, Krane V. Results of the 4D study: ten years of follow-up. Clin Exp Nephrol. 2013. Published online: 29 october.

32. Holdaas H, Holme I, Schmieder RE, Jardine AG, Zannad F, Norby $\mathrm{GE}$, et al., on behalf of the AURORA study group. AT Rosuvastatin in diabetic hemodialysis patients. J Am Soc Nephrol. 2011;22:1335-41. 